\title{
An integrate and fire neural network to simulate epileptic patterns in intracortical EEG
}

\author{
M. Ursino, G.-E. La Cara \& L. Carozza \\ Department of Electronics, Computer Science and Systems, \\ University of Bologna, Italy
}

\begin{abstract}
Epilepsy is characterized by paradoxical patterns of neural activity, either localized within single regions of the cortex or spreading to large areas. These patterns may cause different types of EEG, which may dynamically change during the temporal evolution of seizure. It is generally assumed that these epileptic patterns may originate in a network of strongly interconnected neurons, in cases when excitation dominates over inhibition. The aim of this work is to use a neural network of integrate and fire neurons to analyze which parameter alterations (either at the neural level or at the level of synapses) may induce network instability and epileptic-like discharges. A signal representative of EEG is simulated by summing the membrane potential changes of all neurons.

Model simulations show that an increase in the strength and in spatial extension of excitatory synapses vs. inhibitory synapses and/or an increase in the relative refractory period, may determine sustained patterns of uncontrolled activity, which propagate along the network. These propagating waves may have a different shape and frequency, depending on the particular parameter set used during the simulations and on initial random conditions. The resulting model EEG signals include isolated or repeated bursts, high-frequency low-amplitude waves or larger oscillations with lower frequency. A derangement in a few parameters of the model causes the transition from one pattern to another, thus generating a highly non-stationary signal which resembles that observed during intracortical EEG measurements in epilepsy. The obtained results may help to elucidate the mechanisms at the basis of some epileptic discharges, and to relate rapid changes in EEG patterns, often observed during seizure, with the underlying alterations at the neural and network levels.
\end{abstract}

Keywords: epilepsy, neural models, integrate and fire neurons, EEG. 


\section{Introduction}

Focal seizures of human epilepsy are characterized by well-defined events of electrical activity (cortical EEG), which may rapidly change with time, especially at the onset of seizure. These events may include low-amplitude highfrequency patterns in the $\gamma$-band $(20-100 \mathrm{~Hz})$, high-amplitude low-frequency waves, sporadic spikes or multiple spikes [1,2]. Such phenomena are wellobservable in depth EEG-signals recorded using intracerebral electrodes during surgery. It is generally assumed that the individual patterns, and their temporal evolution during seizure (or even before seizure onset) may provide important information to characterize the epileptic phenomenon and to gain a deeper insight into the underlying physiological mechanisms. Although many studies using signal processing methods have appeared recently to describe intracerebral EEG during epilepsy, just a few works try to investigate the main mechanisms at the neural network level.

A widespread idea is that epileptic seizures in a network of neurons originate from excessive mutual excitation, not sufficiently counterbalanced by interneuron inhibition $[3,4]$. This assumption is at the basis of most recent neural models of epilepsy.

Recently, Wendling et al. [2], using a "neural mass model" of interactions among four neural groups, demonstrated that realistic EEG activities, similar to those observed during seizure, can be produced by modifying a few parameters which establish the strength of synaptic connections among excitatory and inhibitory populations. Neural mass models, however, fail to account for an important characteristic of neural dynamics, i.e., the strong integration of temporal and spatial aspects. Experimental studies demonstrated that networks of neurons can exhibit several spatio-temporal modes of activity: oscillations, synchronism, waves or avalanches $[5,6,7]$. Some of these patterns have been obtained via computer simulations too using network models [8,9,10]. It is thought that these modes correspond to different kinds of behaviour, either of physiological relevance (for instance synchronism) or pathological (such as epilepsy). Cervin et al. [6] in a pivotal study, observed that activity in epileptic cortical slices propagates in a wave-like manner. Milton et al [11] proposed that spiral waves may occur in cortical slices during a paradoxical activation, such as epilepsy.

The previous theoretical and experimental studies suggest that epileptic seizure may be characterized by patterns of activity waves, which propagate along cortical slices in different modes, depending on a balance between excitation and inhibition. We may speculate that, in normal conditions, when inhibition is strong enough to avoid propagation of activity toward surrounding neurons, activity remains confined in a small cortical zone, or induces synchronized activity among functionally interconnected zones. However, in case of insufficient inhibition or stronger excitation, activity waves can originate, which may correspond to different modes of voltage and frequency distribution of power spectral density detected via intracortical EEG. 
The aim of this study is to investigate this problem, using a network of spiking neurons connected via reciprocal excitatory and inhibitory synapses. We wish to analyze how possible alterations in synaptic activity may induce uncontrolled propagating waves, and how these waves may be related with EEG patterns during seizure. The final aim is to reach a deeper insight into the possible relationship between synaptic alterations, patterns of propagating waves in the cortex, and electrical activity recorded via intracortical EEG.

\section{Model description}

The present model is based on the fundamental equations describing the classical Leaky Integrate and Fire (LIF) neuron, with the introduction of some original improvement. In particular, we have provided a description of dynamic for the excitatory and inhibitory synaptic conductance, on the basis of "conductance based models" description, and we have introduced an hyperpolarizing conductance in order to provide a description for the refractoriness property of the neuron.

In the present model, we consider an array of $50 \times 50$ interconnected neural units. The output of the single neural unit is represented by the membrane potential while the overall output network is represented by the mean membrane potential in order to obtain a quantity comparable with the EEG signals commonly recorded.

The network input is represented by a constant depolarizing current injected in a little cluster of neurons. In this way the neurons in the depolarized group fire in synchrony emulating an epileptic focus. Moreover, all neurons receive input Gaussian noise.

We present the network model starting from the single neural unit description.

\subsection{Single neural unit}

Our model is a derivation of the classical LIF model, with a synaptic description following the formalism of cinematic models [12]. The LIF model reduces drastically the complexity in the description of stereotyped behaviour of a single neuron, by replacing the exact dynamic description of the ionic channels involved in the generation of the action potential, with a mechanism thresholdlike.

The classical equations for the LIF model, which provides the action potentials as stereotyped events, can be written as follows:

$$
\begin{cases}\tau_{m} \frac{d V_{m}(t)}{d t}=E_{L}-V_{m}-R_{L} \sum_{s} g_{s}\left(V_{m}(t)-E_{s}\right)-R_{L} g_{t r}\left(V_{m}(t)-E_{t r}\right)+ \\ +R_{L} I_{s t i m} & \text { if } V_{m}(t)<V_{t h} \\ V_{m}(t)=V_{m, \text { max }} & \text { if } t_{\mathrm{j}}<t<t_{\mathrm{j}}+T \\ V_{m}(t)=V_{m 0} & \text { if } t_{\mathrm{j}}+T<t<t_{j}++T+T_{r}\end{cases}
$$


where $V_{m}$ is the membrane potential, $\tau_{m}=R_{L} C_{m}$ is the membrane time constant, $R_{L}$ is the leakage resistance, $I_{\text {stim }}$ represents an external current, $E_{L}$ is the membrane potential at equilibrium, in the absence of synaptic input (often named the leak reversal potential), $V_{t h}$ is a fixed threshold for the spike generation, $t_{j}$ is the instant of the $j$-th spike, $T$ and $T_{r}$ are the duration of the spike and the absolute refractoriness period. $g_{S}$ and $E_{s}$ represent synaptic conductances and their effective reversal potential, while $g_{t r}$ and $E_{t r}$ denote after hyperpolarizing conductance and its reversal potential. In this paper, two kinds of synaptic conductances are included, excitatory ( $\operatorname{subscript} s=e$ ), and inhibitory $(s=i)$.

The previous equations can be explained as follows:

i) the model behaves as a leaky integrator until the membrane potential reaches a threshold, then it generates a spike of duration $T$. During the spike, membrane potential is maintained at a high value, $V_{m, \max }$;

ii) After the spike generation, membrane potential is forced at the resting level, $V_{m 0}$, to simulate the absolute refractory time $\left(T_{r}\right)$. Afterwards the membrane potential is again described by the input integration behaviour.

Moreover, in order to have a clear representation of spiking times, we considered a further quantity for each neuron:

$$
y(t)=\left\{\begin{array}{lr}
1 & \text { if } t_{j}<\mathrm{t}<\mathrm{t}_{\mathrm{j}}+\mathrm{T} \\
0 & \text { otherwise }
\end{array}\right.
$$

i.e., the quantity $y$ is Boolean in type, and assumes value 1 only during the occurrence of a spike.

As specified above, in the model, two different kinds of synaptic conductances are included: i.e., excitatory and inhibitory.

The dynamic of excitatory and inhibitory conductances is described according to a first order kinetic scheme. This scheme describes the interaction between neurotransmitter molecules and postsynaptic receptors, as already proposed in the literature for complex conductance-based neural models [13]. Accordingly, the conductance $g_{S}$ is described as follows

$$
g_{s}=g_{s, \max } P_{s} \quad s=e, i
$$

where $g_{s, \max }$, is the conductance when all ionic channels are open (maximal conductance) and $P_{s}$ is the probability of having an open channel. $P_{S}$ is described with the following well-known kinetic equation

$$
\frac{d P_{s}(t)}{d t}=\alpha_{s}\left(1-P_{s}\right)-\beta_{s} P_{s}
$$

where $\alpha_{s}$ is the opening rate, $\beta_{s}$ the closing rate of the channels.

In order to account for the effect of excitatory and inhibitory influences, coming from other neurons in the network, we assumed that the opening rate in Eqs. (4) depends on a synaptic input (say $S$ with $S=E x$ and $S=I n$ as to excitatory and inhibitory inputs, respectively). The latter describe the overall influence of other neurons in the network, as described below. Eq. 5 can be rewritten as follows: 


$$
\tau_{s}(S) \frac{d P_{S}(t)}{d t}=P_{s}^{\infty}(S)-P_{s}(t)
$$

where:

$$
\begin{gathered}
\tau_{s}(S)=\frac{1}{\alpha_{s}(S)+\beta_{s}} \\
P_{s}^{\infty}(S)=\frac{\alpha_{s}(S)}{\alpha_{s}(S)+\beta_{s}}
\end{gathered}
$$

with $0 \leq P_{s}^{\infty}(S) \leq 1$.

In order to describe the influence of synaptic input on the opening rate, we assumed that, in the absence of the synaptic input, the opening rate is zero. The higher the synaptic input, the higher the opening rate, up to a maximum saturation level. This is described with the following equation:

$$
\alpha_{s}(S)=\alpha_{s, \max }\left(1-e^{-S / k_{s}}\right)
$$

where $\alpha_{s, \max }$ is the saturation for the opening rate, and $k_{s}$ is a constant which establishes the slope of the sigmoidal relationship.

A further aspect in the description of a single neuron concerns the presence of a hyperpolarization conductance, named $g_{t r}$, which simulates the relative refractoriness period and the phenomenon of "spike rate adaptation". The conductance $g_{t r}$ assumes its maximum value $g_{\text {thighr }}$ after the spike generation, maintains this value throughout the absolute refractory period (i.e., $t_{j}<t<t_{j}+T$ $+T_{r}$ ) and then falls to zero accordingly to the following equation:

$$
\tau_{g t r} \frac{d g_{t r}(t)}{d t}=-g_{t r}(t)
$$

\subsection{Synaptic connections}

The previous description concerns a single neuron. However, in a network neurons communicate via the synaptic terms, $S=E x$ and $S=I n$ in Eqs. 4-8. In order to describe a network of neurons, arranged in a regular lattice, in the following the quantities in each neuron will be represented using two subscripts, $i$ and $j$, which denote the row and column position of the neuron.

Of course, various different arrangements for the synapses are possible, depending on the particular network. In the present study we assumed a classical Mexican hat disposition for the synapses, i.e., one neuron receives excitatory and inhibitory synapses from a layer of proximal neurons, with excitation having narrower extension than inhibition. Moreover, we assumed that the strength of the synaptic lateral connections among neurons in the network decreases with the distance, named $d$, between the pre and postsynaptic neurons. Furthermore, in order to account for the intrinsic variability of synapses, we assumed that excitation follows a random uniform distribution, between 0 and a maximum value. This signifies that not all neurons really receive strong excitation, but only 
a portion of them chosen randomly. Hence, the following equations can be written for terms Ex and In of the neuron $i, j$.

$$
\begin{aligned}
& E x_{i j}=\sum_{l, m=1}^{N} W_{e x 0} \cdot \rho_{i j} \cdot e^{\frac{d_{i j, l m}{ }^{2}}{\sigma_{e x}^{2}}} \cdot y_{l m} \\
& I n_{i j}=\sum_{l, m=1}^{N} W_{i n 0} \cdot e^{\frac{d_{i j, l m}^{2} \sigma_{i n}^{2}}{\sigma^{2}} \cdot y_{l m}} \\
& d_{i j, l m}=\sqrt{(i-l)^{2}+(j-m)^{2}}
\end{aligned}
$$

where $W_{e x 0}$ and $W_{i n 0}$ are the strength of excitatory and inhibitory synapses, respectively; $\sigma_{e x}, \sigma_{i n}$ are the standard deviations, and $\rho_{i j}$ represents a random variable with uniform distribution between 0 and 1 .

The contribution of excitatory and inhibitory synapses described above affects the dynamic of synaptic conductances and then the currents crossing the membrane, with a consequent modulation in the membrane potential evolution.

Finally, the output from the overall network is computed as the mean value of membrane potential of all neural units in the network. In this way it is possible to obtain a quantity comparable with the commonly recorded EEG clinical signal. By denoting $V_{E E G}(t)$ this output, we have:

$$
V_{E E G}(t)=\frac{\sum_{i=1}^{N} \sum_{j=1}^{N} V_{m, i j}(t)}{N \cdot N}
$$

All parameters of the model have been taken according to physiological considerations. They are not reported here due to space limitation.

\section{Results}

In the following, just a few examples of the possible variability of results are presented, for the sake of brevity. A first set of simulations has been performed by increasing parameter $W_{e x 0}$ in Eq. 10 from an initial low value, up to a higher value which induces strong instability and the appearance of propagating waves.

When $W_{\text {exo }}$ has a low value (about 0.1 or 0.2 ) the activity in the network remains confined within the small central focus, without propagating toward adjacent neurons. This results in a quite constant small amplitude potential in Eq. 11. The effect of increasing parameter $W_{\text {exo }}$ is depicted in Fig. 1. First, when $W_{\text {ex } 0}=0.35$ (see panels a)), one can observe the occurrence of small sporadic waves which propagate slowly along the network, and, at certain instants, may join together to form large wave fronts. This behaviour results in the appearance of some large amplitude waves, with a low frequency content. When $W_{\text {exo }}$ is further increased up to 0.6 (panel b)), we have the initial formation of circular waves, which propagate form the central focus to the periphery. This phase 
corresponds to the occurrence of small amplitude high frequency oscillations in the average potential. However, at certain instants (around $300 \mathrm{~ms}$ in panel b)) these waves may break down into different separate waves. This corresponds to the appearance of larger oscillations in the EEG, in part similar to multiple spikes. At higher values of $W_{\text {exo }}(1.2$ panel c)) we have only circular regular waves, which propagate rapidly across the network. In this condition, the average potential exhibits small amplitude high-frequency oscillations. Finally, if excitation is further raised $\left(W_{\text {ex } 0}=1.5\right.$, panel $\left.\mathrm{d}\right)$ ), circular waves may break down again, resulting in irregular waves, with a wide frequency content. The previous examples show the great number of behaviours which may originate in the network even from changes in a single parameter only.

a)

b)

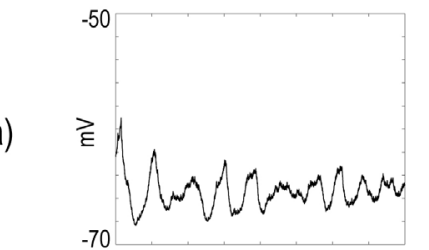

c)

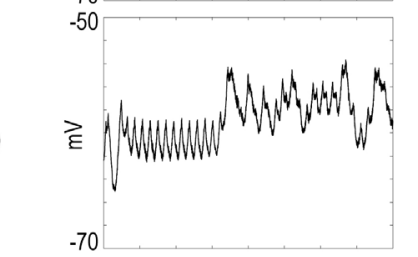

d)

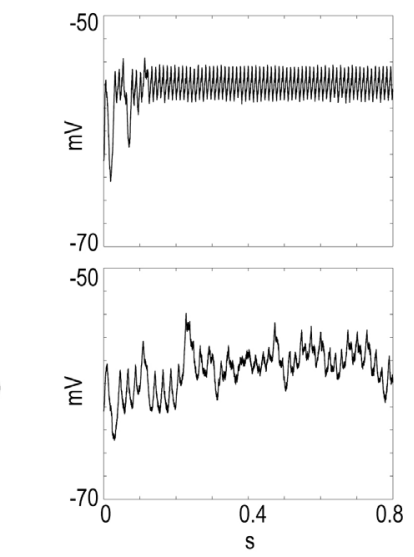

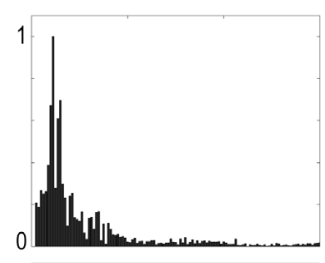
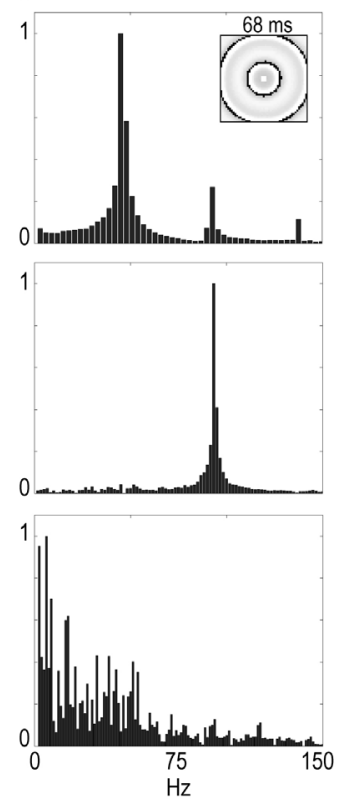
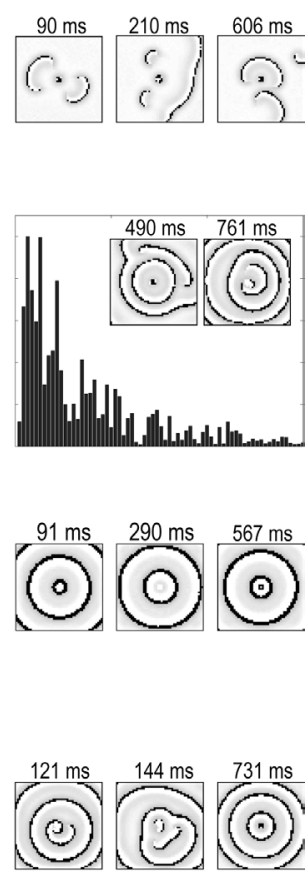

Figure 1: Sensitivity analysis on the role of strength of excitatory synapses (parameter $W_{e x 0}$ ). In each row the first left panels represent the simulated EEGs, the middle panels show the amplitude spectra, the right little panels represent the output of neurons at different time steps in the simulation. In row b) two amplitude spectra are presented: the snapshots of network activity are enclosed in the relative spectrum panel. a) $W_{e x 0}=0.35$; b) $W_{e x 0}=0.6$; c) $W_{e x 0}=1.2$; d) $W_{e x 0}=1.5$. 
A second example is illustrated in Fig. 2. This figure shows the average membrane potential, and some snapshots of network activity, obtained with $W_{\text {exo }}$ $=0.8$, by increasing the time constant $\tau_{\text {gtr }}$ in Eq. 9 from its previous value $(15 \mathrm{~ms})$ to $30 \mathrm{~ms}$. This signifies an increase in relative refractory period and in spike adaptation. Average membrane potential exhibits high-frequency oscillations (which correspond to circular waves) interrupted by some isolated spikes.
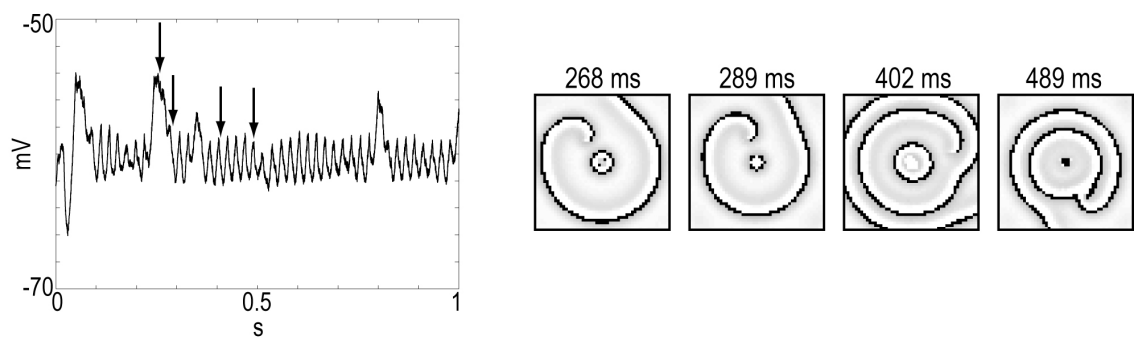

Figure 2: The effect of increasing parameter $\tau_{\mathrm{gtr}}$ related with relative refractoriness period and spike adaptation phenomenon. $\tau_{\mathrm{gtr}}$ in this simulation is equal to $30 \mathrm{~ms}$, vs. $15 \mathrm{~ms}$ of basal value.

\section{Discussion}

Paradoxical events during epilepsy are characterized by specific patterns of intracortical EEGs, which may change rapidly with time in a highly nonstationary way $[1,2]$. Objective of this work was to investigate how epileptogenic patterns may emerge in a small network of integrate and fire neurons, as a consequence of an impaired balance between excitation and inhibition, joined with a randomized distribution of synapses. The choice of a random distribution of synapses is justified by the assumption that they reflected previous learning episodes stored in the network, for instance, previous correlation between presynaptic and postsynaptic activity.

Simulation results demonstrate that the network can generate different patterns of average potential, including low-frequency irregular waves with large amplitude, small-amplitude high-frequency oscillations, or even spikes. An important new contribution, not addressed in previous studies, is the relationship between these patterns and the different modalities of propagating waves. We observed that small erratic waves, which can almost vanish at some instants of the simulation, or join with other waves at other instants to form large wave fronts (see Fig. 1, panel a) ) cause low frequency signals in the EEG, whose amplitude may exhibit large variations. Circular waves, which propagate quickly from the focus to the periphery, can induce oscillations with higher frequency but small constant amplitude (Fig. 1 panel c)). A rupture of these waves, however, may induce more complex patterns of activity, with a wider frequency content (see the second part of panel $\mathrm{c}$ in Fig. 1), which may also change 
abruptly with time. It is worth noting that just a small portions of the simulations performed with the model are reported in section results, for the sake of brevity. Similar patterns, but with a greater richness of behaviour, have been observed not only by increasing the strength of excitatory synapses, but also reducing the strength of inhibitory synapses, or modifying the extension of the "Mexican hat" (i.e., by varying the standard deviations $\sigma_{e x}, \sigma_{i n}$ in Eq. 10). The role of parameter $\tau_{g t r}$, which is related with the refractoriness period and self-adaptation, is also important and seems to play a significant role in the generation of spikes (i.e., in causing a large burst of activity followed by a period of depressed activity, see Fig. 2). These results underline the complexity of behaviours which can be obtained with the network, by modifying just a few parameters and provide important indications on the possible relationships between the temporal characteristics of intracortical EEG (its shape and frequency content), the spatial distribution of propagating waves, and the underlying neural mechanism at the synaptic and cellular level.

Finally, we remind that, in the present work we assumed that the synaptic strength always decreases with the distance between the presynaptic and postsynaptic neuron. This choice produces, as a natural consequence, the formation of waves which tend to propagate towards adjacent neurons. Of course, different dispositions of synapses could also be assumed, which may induce alternative behaviours. For instance, the use of sparse but strong synapses, which link remote neurons, have been investigate by others: these networks exhibit a variety of states too, including synchronization and asynchronous states $[9,10]$. Another possibility comprises the so-called "small world" disposition [14]: neurons are especially linked to adjacent units (as in the present work) but some neurons send strong synapses to remote zones. Finally, it has been suggested that a branching disposition of synapses may lead to a different mode of propagation, named "avalanche" [7]. The effect of these alternative patterns of connectivity on network behaviour, and their implication in paradoxical (epileptogenic) discharges may deserve future work.

\section{References}

[1] Ebersole, J.B. \& Milton, J. The electroencephalogram (EEG): a measure of neural synchrony, in Epilepsy as a dynamical disease, Milton, J. \& Jung, P. Ed. Berlin: Springer-Verlag, ch. 5, pp. 51-68, 2003.

[2] Wendling, F., Bartolomei, F., Bellanger, J.J. \& Chauvel, P., Epilepsy fast activity can be explained by a model of impaired GABAergic dendritic inhibition. Eur. J. Neurosci., 15(9), pp. 1499-1508, 2002.

[3] Dichter, M.A. Basic Mechanisms of epilepsy: targets for therapeutic intervention. Epilepsia, 38, pp. S2-S6, 1997.

[4] Cossart, R., Dinocourt, C., Hirsch, J.C., Merchan-Perez, A., DeFelipe, J., Ben-Ari, Y.M., Esclapez, M., \& Bernard, C., Dendritic but not somatic GABAergic inhibition is decreased in experimental temporal lobe epilepsy. Nature Neurosci., 4, pp. 52-62, 2001. 
[5] Chervin, R.D., Pierce, P.A., Connors, B.W., Periodicity and directionality in the propagation of epileptiform discharges across neocortex. $J$. Neurophysiol., 60, pp. 1695-1713, 1988.

[6] Engel, A.K., Singer, W., Dynamic predictions: oscillations and synchrony in top-down processing. Nat. Rev. Neurosci., 2, pp. 704-716, 2001.

[7] Beggs, J.M., Plenz, D., Neuronal avalanches in neocortical circuits. The J. of Neurosci., 23(35), pp. 1167-1177, 2003.

[8] Jefferys, J.G., Traub, R.D., Whittington, M.A., Neuronal networks for induced '40 Hz' rhythms. Trends Neurosci., 19(5), pp. 202-208, 1996.

[9] Brunel, N., Dynamics of sparsely connected networks of excitatory and inhibitory spiking neurons. J. Comput. Neurosci., 8(3), pp. 183-208, 2000.

[10] Kudela, P., Franaszczuk, P.J., Bergey, G.K., Changing excitation and inhibition in simulated neural networks: effects on induced bursting behavior. Biol. Cybern., 88(4), pp. 276-85, 2003.

[11] Milton, J.G., Chu, P.H., \& Cowan, J.D., Spiral waves in integrate-and-fire neural networks. Advances in Neural information processing systems, Hanson, S.J., Cowan, J.D., \& Giles, C.L., eds., vol. 5, pp. 1001-1007, 1993.

[12] Dayan, P., \& Abbott, L.F., Theoretical neuroscience computational and mathematical modelling of Neural Systems. The MIT press, 2001.

[13] Destexhe, A., Mainen, Z. F., \& Sejnowski, T. J. An Efficient Method for Computing Synaptic Conductances Based on a Kinetic Model of Receptor Binding. Neural Computation, 6, pp. 14-18, 1994.

[14] Watts, D.J., Strogatz, S.H. Collective dynamics of 'small-world' networks. Nature, 393(4), pp. 440-442, 1998. 